\title{
The design of remote traffic light control system using Raspberry-Pi
}

\author{
Van Anh Nguyen, Duy Khanh Pham, Van Nam Bach, Phuong Huy Nguyen
}

\begin{abstract}
The traffic light system has been working based on the specific signal light timing diagram, in wich, the time period for each lane is unchangeable. In some situation, the number of vehicles at each lane is much different, specifically, there are not any or so few vehicles at one lane, but there are so many vehicles at the others, and the time periods for all the lanes are still the same. This leads to many vehicles have to wait in unnecessary periods.

In this paper, authors proposed a method to design a remote-control system for the traffic light which flexibly controls time periods through camera system. Raspberry-Pi, functions as the central process unit, both sends the image to Traffic Management Center and provides the traffic signals for traffic light system. This method is evaluated through the real experimental model.
\end{abstract}

Index Terms - traffic light control system, vehicles, camera system, Raspberry-Pi.

\section{INTRODUCTION}

Vietnam, a developing country in transition, has an estimated population density of 274 people per square kilometer, 5.2 times bigger than the world's population. In addition, the situation of over-urbanization in Vietnam has led the growing numbers of people moving into cities. As a result, cities face big issues: housing, environment, jobs, health care ... especially, traffic. The development of transport system is not catching up the increment of vehicles, the traffic congestion constantly happens on the main roads, these cause negative affects in daily life.

There have been a lot of solutions to solve these problems, one of the most popular solutions is using the smart camera to determine the traffic density, and automatically adjust the time for each lane [1], [2]. However, this method has its limitations, because it requires a high-resolution camera, complex algorithm, and the accuracy of obtained results depends to the weather conditions. In addition, this is the automatic system, it is always required for high reliability. From this, authors proposed an approach to remotely control the traffic light, in which, human plays the key role in deciding the operation of system. The normal operation of the traffic light is based on available signal light timing. When there is a significant difference in the traffic density at lanes (such as, the traffic density at lane 1 is much higher than lane 2 ), the traffic management center operator will remotely made

Van Anh Nguyen, Student of Faculty of Electronics, Thai Nguyen University of Technology, Thai Nguyen, Vietnam.

Duy Khanh Pham, Department of Electronics Engineering, Tha Nguyen University of Technology, Thai Nguyen, Vietnam, +84972992282

Van Nam Bach, Department of Electronics Engineering, Thai Nguyen University of Technology, Thai Nguyen, Vietnam, +84936665822

Phuong Huy Nguyen, Department of Electronics Engineering, Thai Nguyen University of Technology, Thai Nguyen, Vietnam, +84912488515 necessary adjustments for the signal light timing for traffic light via website.

\section{SURVEY TRAFFIC LIGHT SYSTEM CROSSROADS AND SYSTEM DIAGRAM}

As analyzed above, project proposed the method to remotely control the traffic light system, the authors investigated some intersections near Thai Nguyen university of techonology (Figure 1), traffic flow diagram is provided in Figure 2.

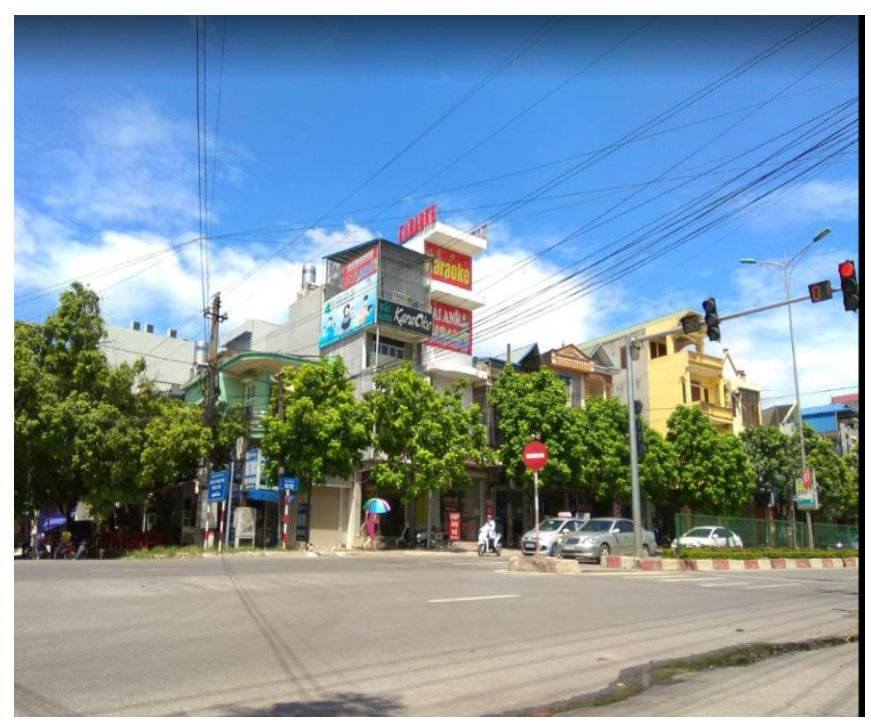

Fig. 1: The intersection

Traffic lights at this intersection include:

- There are 4 lampposts.

- Light time of light:

$+\operatorname{Red}=45 \mathrm{~s}$, Light $=3 \mathrm{~s}$, Lightning $=42 \mathrm{~s}$ in peak mode;

+ Red $=30 \mathrm{~s}$, Yellow $=3 \mathrm{~s}$, Txanh $=27 \mathrm{~s}$ in low light mode;

+ and Yellow only in Free mode.

- Displays countdown time by matrix LED.

- Include three signal lights Green, Red, Yellow.

- Works in three modes: Peak, Low and Free

- There is no lane allocation at all times.

- Have a left turn first.

The operation principle of the traffic light at the intersection as follows: in low light mode, the light at point $\mathrm{A}$ and $\mathrm{C}$ turn green (for $27 \mathrm{~s}$ ), allowing vehicles to move in two directions from A to $\mathrm{C}$, and $\mathrm{C}$ to A. Simultaneously, the light at point $\mathrm{B}$ and $\mathrm{D}$ turn red (for 30s), prohibit vehicles from moving from $\mathrm{B}$ to $\mathrm{D}$, and $\mathrm{D}$ to $\mathrm{B}$. After a period of time $(27 \mathrm{~s})$, the light at point $\mathrm{A}$ and $\mathrm{C}$ change to yellow (for $3 \mathrm{~s}$ ). Then, the light at point $\mathrm{A}$ and $\mathrm{C}$ turn red, the light at point $\mathrm{A}$ and $\mathrm{C}$ simultaneously turn green. This process is iterative. This diagram below illustrates the operation of the traffic light. 


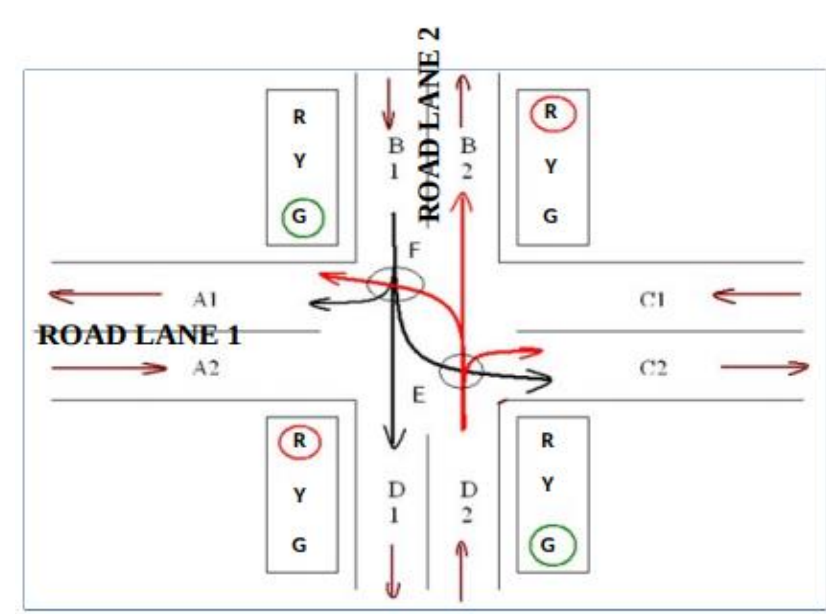

Fig. 2: The traffic flow diagram

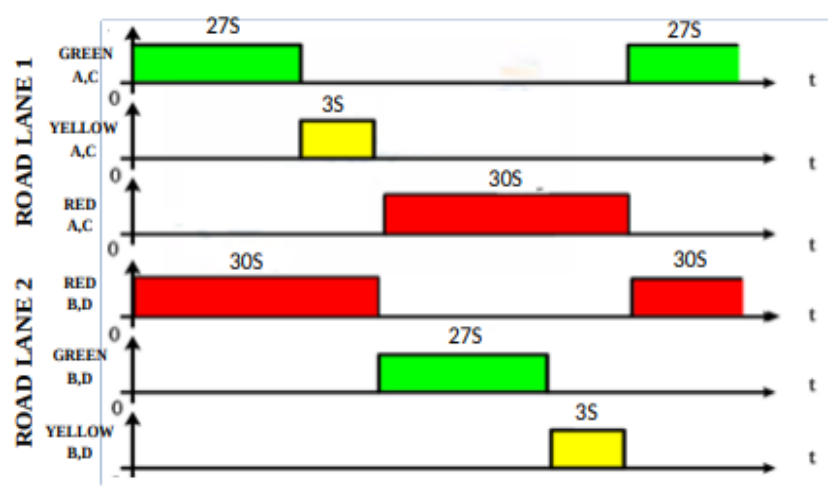

Fig. 3: The timing diagram for the traffic light system in Figure 2

Obiviously, the signal light timing for this traffic light is unchangeable and independent of the traffic density. To solve this problem, authors proposed a block diagram of the traffic light system as shown in Figure 4.

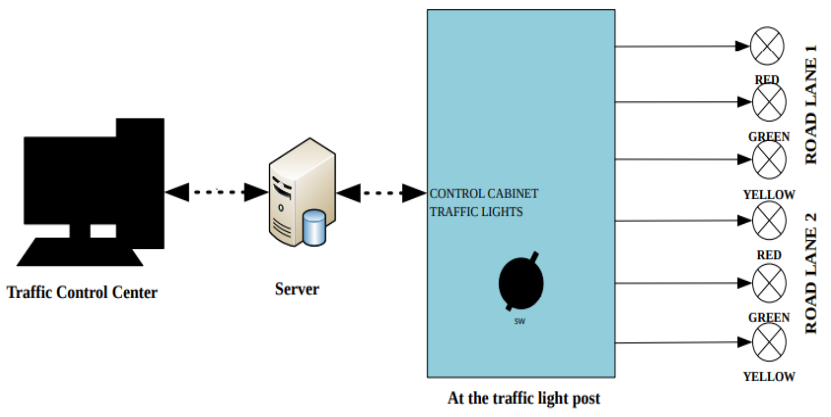

Fig. 4: Block diagram of system

The working priciple of system: At normal condition, the operation of system is based on the timing diagram in Figure 3 , when the difference in traffic density between two lanes occurs, the traffic management center operator will set the new signal light timing for the traffic light system control cabinet through the server. The central processing unit of control cabinet is Raspberry Pi.

\section{DESIGN OF SYSTEM}

\subsection{Raspberry Pi 3}

Raspberry $\mathrm{Pi}$ is a series of small single-board computers developed in the United Kingdom by the Raspberry Pi
Foundation, and Raspberry Pi 3 is the latest version of the Raspberry Pi family. Raspberry Pi 3 is designed with CPU ARM Cortex-A53 Quadcore 1.2GHz 64-bit, RAM 1GB, and supports Wifi 802.11n and Bluetooth 4.1. Raspbian operating system is updated to the latest version for 64-bit operation [3].

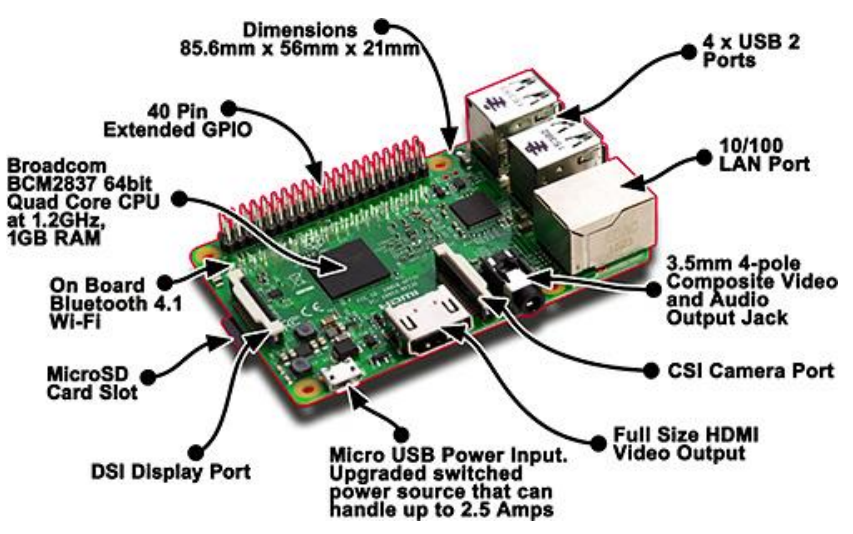

Fig. 5: Raspberry Pi 3

At a fraction of the cost, compact size and functionality, Raspberry is well suited for inclusion in the academic and research agenda of schools.

Specifications:

- Broadcom BCM2837 chipset running at $1.2 \mathrm{GHz}$

- 64-bit quad-core ARM Cortex-A53

- $802.11 \mathrm{~b} / \mathrm{g} / \mathrm{n}$ Wireless LAN

- Bluetooth 4.1 (Classic \& Low Energy)

- Dual core Videocore IV® Multimedia co-processor

- 1 GB LPDDR2 memory

- Supports all the latest ARM GNU/Linux distributions and Windows 10 IoT

- MicroUSB connector for 2.5 A power supply

- 1 x 10/100 Ethernet port

- 1 x HDMI video/audio connector

- 1 x RCA video/audio connector

- 4 x USB 2.0 ports

\subsection{Simulation of system using Proteus}

The real model for system is designed as shown in Figure 7, and Proteus software is used to simulate the operation of the system as shown in Figure 6.

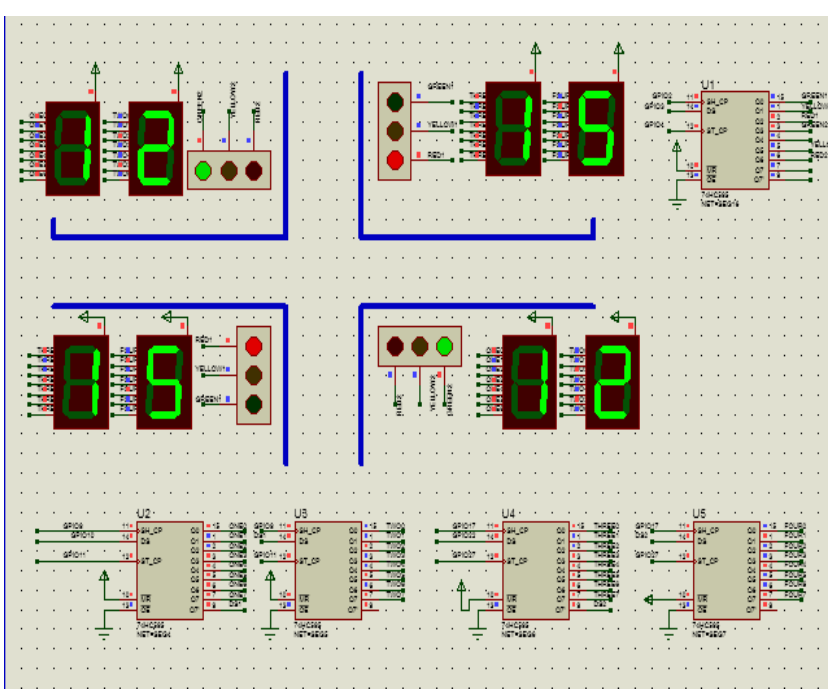

Fig. 6: Simulation of system 


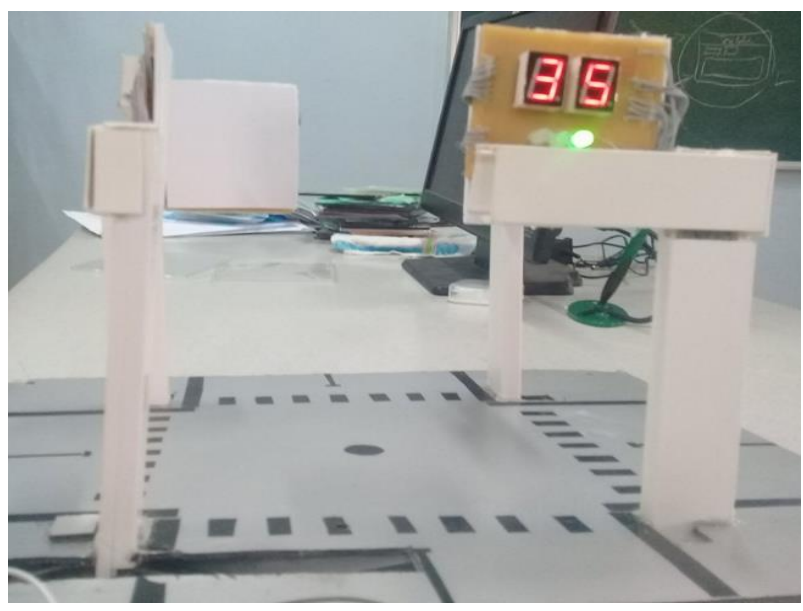

Fig. 7: Model of traffic light system

\subsection{Software}

The traffic light system control website is based on ASP.NET, an open-source server-side web application framework, designed for web developer to produce dynamic web pages, web applications, and web services. The interface of the traffic light system control website is shown in Figure 8. Through the website, the operator can set two operation modes for the traffic light system: The daytime operation mode and nighttime operation mode. Also, through the website, operator can set the traffic signal timing for the traffic light system corresponding to the change of traffic density at the intersection.

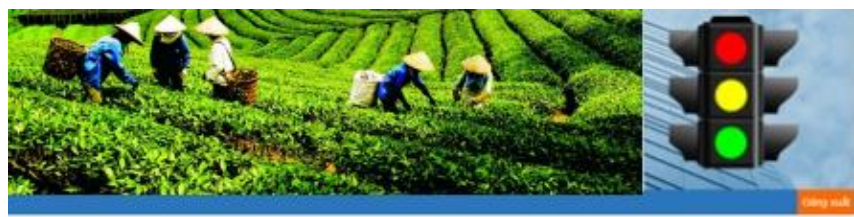

List of traffic lights in the province of Thai Nguyen

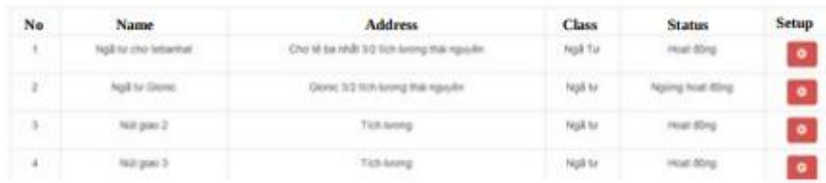

Fig. 8: The traffic light system control website interface

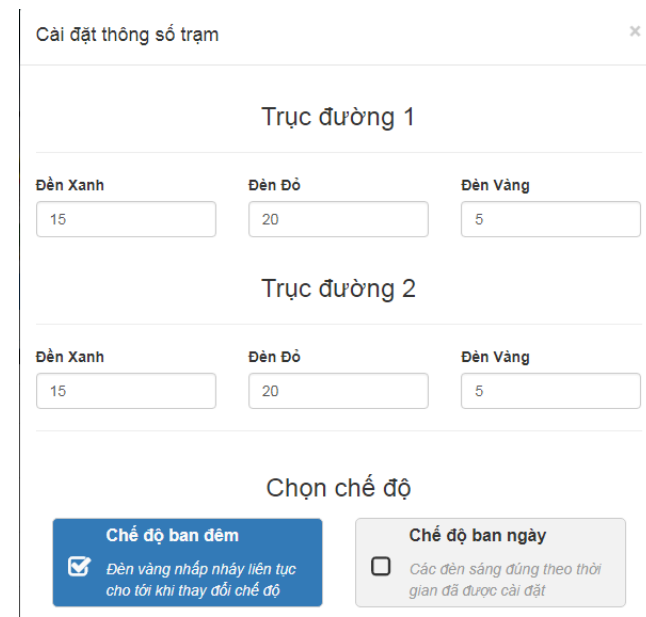

Fig. 9: Parameter setting interface

\section{CONCLUSION}

With this project, authors proposed a method to remotely control traffic light system. This method will help to decrease waiting time at red light, to aviod traffic jams. The experimental model, the control website are designed to evaluate the operation of system. However, with this method, the controlled intersection is just selected manually (by operator), the future plan for this project is applying the automatic image processing algorithm to select the controlled intersection, which is desirable for incresing the speed of the system.

\section{ACKNOWLEDGMENT}

This research was supported by a grant for the university research from the TNUT (Thai Nguyen University of Technology), under project T2017-BS03. We thank our colleagues from the TNUT who provided insightand expertise that greatly assisted the research.

\section{REFERENCES}

[1] Alex, "Traffic Light Control System Using Image Processing", International Journal of Innovative Research in Computer and Communication Engineering (An ISO 3297: 2007 Certified Organization) Vol.2, Special Issue 5, October 2014.

[2] Ms.Pallavi Choudkalet. al, "Real time traffic light control using imageprocessing", Indian journal of Computer Science and Engineering(IJCSE) ISSN:0976-5166, Vol 2, No.1,pp 6-10.

[3] https://vi.wikipedia.org/wiki/Raspberry_Pi.

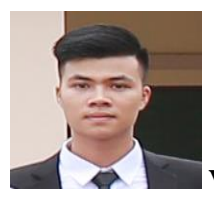

Van An Nguyen is student of Faculty of Electronics, Thai Nguyen University of Technology, Thai Nguyen, Vietnam. Research interests: Analog electronics, Digital electronics, Microprocessor and Microcontroller.

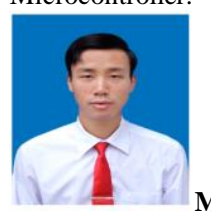

Semiconductor Device Polytechnic Institute", Ukraine. He is working at Faculty of Electronics Engineering, Thai Nguyen University of Technology, Thai Nguyen City, Vietnam. Research interests: Semiconductor materials, Analog electronics, Digital electronics, Biomedical electronics.

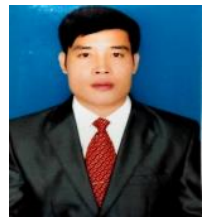

MSc Van Nam Bach is master in Electronic engineering at Thai Nguyen University of Technology, Viet Nam. He is working at Faculty of Electronics Engineering, Thai Nguyen University of Technology, Thai Nguyen City, Vietnam. Research interests: Analog electronics, Digital electronics, Design Printed Circuit Board.

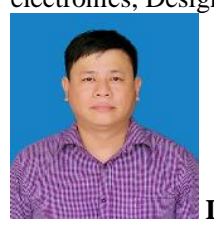
Engineering at Hanoi University of Technology, Vietnam. He is working at Faculty of Electronics Engineering, Thainguyen University of Technology, Thai nguyen City, Vietnam. Research interests: Digital Signal Processing, Soft computing, Fuzzy control. 